\title{
Infecciones de Transmisión Sexual en un Grupo de Alto Riesgo de la Ciudad de Montería, Colombia
}

\author{
Sexually-transmitted infection in a high-risk group from Montería, \\ Colombia
}

Nelson Alvis' ${ }^{1}$ Salim Mattar², Jair Garcia², Edwin Conde ${ }^{2}$ y Alberto Diaz ${ }^{2}$

1. Departamento de Investigaciones Económicas y Sociales, Universidad de Cartagena, Colombia.nalvis@yahoo.com

2. Instituto de Investigaciones Biológicas del Trópico, Universidad de Córdoba. mattarsalim@hotmail.com, garciajair@hotmail.com, bladex@hotmail.com, albertods@hotmail.com

Recibido 2 Junio 2006/Enviado para Modificación 2 Diciembre 2006/Aceptado 25 Enero 2007

\section{RESUMEN}

Objetivo Identificar los principales agentes etiológicos de enfermedades de transmisión sexual en una población de alto riesgo de la ciudad de Montería.

Métodos La población estuvo conformada por 69 trabajadoras sexuales (grupo de alto riesgo) y 16 amas de casa (grupo de bajo riesgo) de la ciudad de Montería. Las muestras de secreción vaginal fueron procesadas según métodos microbiológicos estándares y por el método molecular de AMPLICOR® CT/NG.

Resultados La edad de las pacientes estuvo entre 18 y 44 años con una media de 26,1 . En la población de alto riesgo, se determinó que el $17,4 \%$ fueron positivas para Gardnerella vaginalis, C. trachomatis (5,9\%); Neisseria gonorrhoeae (4,3\%), Trichomonas vaginalis y Candida albicans, (2,9\%); en la población de bajo riesgo Gardnerella vaginalis $(56,3 \%)$, C. trachomatis (12,5\%); Neisseria gonorrhoeae $(6,3$ $\%)$, y Candida albicans (12,5\%). Se encontró que la frecuencia de relaciones sexuales en la población de trabajadoras sexuales fue: $70 \%$ (5-10 semanales), 10 $\%$ (11-15 semanales) y $20 \%$ (16-20 semanales). El 15,4 \% de las trabajadoras sexuales no utilizaron preservativos en sus relaciones sexuales.

Conclusión Las altas tasas de infección encontradas en las poblaciones estudiadas presumen que existe un alto riesgo de transmisibilidad y es prioridad intervenir en estos grupos para prevenir las infecciones por el VIH y demás infecciones de transmisión sexual

Palabras Clave: Infecciones por transmisión sexual, trabajadoras sexuales, Colombia, Chlamydia trachomatis, Neisseria gonorrhoeae (fuentes: DeCS, BIREME). 


\section{ABSTRACT}

Objective Identifying the main aetiological agents of sexually transmitted infections (STI) in a high-risk population from the city of Montería, Colombia.

Methodology The population consisted of 69 sex-workers (high-risk group) and 16 housewives (low-risk group) living in the city of Montería. Specimens were cultured by standard microbiological methods and by the AMPLICOR CT/NG molecular technique.

Results Patients were aged 18-44 (26.1 average age). It was determined that $17,4 \%$ of the high-risk population were positive for $G$. vaginalis, $15,9 \%$ for $C$. trachomatis, $4,3 \%$ for $N$. gonorrhoeae and 2,9\% for T. vaginalis and Candida albicans and, in the low-risk population, 56,3\% for G. vaginalis, $12,5 \%$ for $\mathrm{C}$. trachomatis, 6,3\% for $\mathrm{N}$. gonorrhoeae and $12,5 \%$ for $C$. albicans. It was found that $70 \%$ of the sex-workers had 5-10 sexual relationships per week, $10 \%$ 11-15 per week and $20 \% 16-20$ per week (average above 1000 annual partners). 15,4\% of the sex-workers did not use protection during their sexual relationships.

Conclusions The high rates of infection found in the populations studied presume a high risk of transmission, making it a priority to intervene in these groups to prevent the spread of HIV and STI.

Key Words: Sexual transmission, sex-worker, Colombia, Chlamydia trachomatis, Neisseria gonorrhoeae (source: MeSH, NLM).

L

as infecciones de transmisión sexual (ITS) son un problema de salud pública en los países en vías de desarrollo debido a la infertilidad, discapacidad a corto plazo y las consecuencias médicas y psicológicas para miles de hombres, mujeres y niños (1-8). Las consecuencias de las ITS son dramáticas si se tiene en cuenta su contribución potencial a la transmisión de la infección por VIH. Los estudios epidemiológicos de vigilancia sobre las poblaciones vulnerables son claves debido a la información que suministran para los programas de prevención e intervención. La OMS (1) y ONU-SIDA consideran que la vigilancia de las ITS es un componente insustituible de los sistemas de vigilancia del VIH-SIDA $(1,3,7)$. OMS (1) considera cuatro elementos cruciales en la vigilancia de las ITS: notificación de casos, medición de la prevalencia y monitoreo, evaluación de la etiología de los síndromes de las ITS y el monitoreo de la resistencia a los antimicrobianos.

De otro lado, las conductas sexuales riesgosas facilitan la transmisión de las ITS y el HIV. Las trabajadoras sexuales son claves para la transmisión de las ITS y del VIH, éste es el grupo centinela con la población general. El control de las ITS entre las trabajadoras sexuales es crucial, ya que es un indicador estimativo de los costos económicos que ocasionan los tratamientos de las ITS tanto en los grupos de alto riesgo como en el de bajo riesgo (9-13). 
La escasez de datos básicos sobre la verdadera incidencia y prevalencia de las ITS, no permite contar con información confiable para estimar el impacto de la transmisión de este tipo de enfermedades en los países en vía de desarrollo como el nuestro. Por ello, se considera importante realizar estudios que provean una aproximación a la prevalencia de ITS en la ciudad de Montería no se conocen estudios en los últimos 50 años sobre la epidemiología de las ITS.

El objetivo de este estudio fue identificar los principales agentes etiológicos de enfermedades de transmisión sexual en una población de alto riesgo de la ciudad de Montería.

\section{MATERIALES Y METODOS}

Tipo de estudio, población y aspectos epidemiológicos

Se realizó un estudio descriptivo de corte transversal para conocer las características epidemiológicas y el diagnóstico microbiológico y molecular de las ITS. El universo poblacional lo constituyeron las 200 trabajadoras sexuales que regularmente asisten a controles al Centro de Atención Médico de Urgencias-CAMU La Granja- de la ciudad de Montería. La muestra la integraron 69 de estas trabajadoras sexuales, seleccionadas de manera consecutiva y no probabilística, quienes decidieron participar en el estudio y a quienes se les denominó Grupo de Alto Riesgo. Por otro lado, como grupo control se tomaron 16 amas de casa, no trabajadoras sexuales, que asistieron a cita médica al centro de salud del barrio P5 a quienes, luego informar los propósitos del estudio y de otorgar su consentimiento voluntario a participar en la investigación, se les denominó Grupo de Bajo Riesgo. A todas las pacientes se le aplicó una encuesta que indago sobre algunos de estos factores de riesgo: i) utilización de preservativos en las relaciones sexuales, ii) antecedentes de ITS, iii) uso frecuente de sustancias antimicrobianas como óvulos, lavados o cremas vaginales, iv) otros datos epidemiológicos y sociodemográficos como edad, tiempo laboral y nivel educativo.

Aspectos microbiológicos e identificación molecular

La toma de muestras se llevo a cabo por métodos estándares previamente establecidos (14). Básicamente, la muestra de secreción vaginal fue recolectada en dos escobillones estériles. Uno fue utilizado para hacer una coloración de Gram, test de aminas y el examen directo detectar de células epiteliales, bacterias, leucocitos polimorfonucleares, eritrocitos, presencia de blastoconidias, 
pseudomicelios y trofozoítos de Trichomonas. Las muestras se transportaron en el medio de Amies y se cultivaron en agar GC suplementado con Vitox ${ }^{\circledR}$ y Vcnt ${ }^{\circledR}$, agar chocolate con suplemento y agar sangre (14) todas las colonias sospechosas de $N$. gonorrhoeae se identificaron (14). El tercer escobillón que contenía medio de transporte AMPLICOR-STD ${ }^{\circledR}$ fue conservado a -70 ${ }^{\circ} \mathrm{C}$, Antes de la identificación molecular, por el método de AMPLICOR ${ }^{\circledR}-\mathrm{CT} /$ NG (Roche, Branchburg, N.J). Esta prueba se basa en cuatro procesos principales: preparación del espécimen, amplificación por PCR del ADN con iniciadores biotinilados, hibridación de los productos amplificados con sondas oligonucleótidas especificas y detección por colorimetría de los productos amplificados y unidos a las sondas. La prueba AMPLICOR CT/NG es un ensayo múltiple que permite la amplificación simultanea del ADN de $C$. trachomatis y $N$. gonorrhoeae.

Análisis de los resultados

Los datos fueron recolectados mediante un formulario estandarizado y tabulados en una hoja electrónica de MS Excel®. Adicionalmente, se utilizaron las herramientas de análisis de datos de este programa para elaborar la estadística descriptiva.

\section{Aspectos éticos}

Este proyecto se rigió de acuerdo a las normas técnicas, científicas y administrativas para la investigación en salud, del Ministerio de Salud de Colombia resolución numero 008430 del 4 de octubre de 1993 y la declaración de Helsinki refrendada del año 2004 (15) El estudio protegió la privacidad e intimidad del individuo identificándolo con un número interno. El proyecto fue aprobado y catalogado por el comité de ética del instituto de investigaciones biológicas del trópico como una investigación de riesgo mínimo, en la cual únicamente se obtuvieron secreciones vaginales. Los datos obtenidos de estos análisis rutinarios y de diagnóstico se incluyeron en una base de datos anónima y se codificó con un número. A todos los sujetos que fueron involucrados en esta investigación se le explico el tipo de estudio y de ellas se obtuvo su consentimiento oral y/o escrito.

\section{RESULTADOS}

La edad de grupo de alto riego estuvo entre 18 y 44 años con una media de 26,1 (I.C. 95 \%: 24,7-27,6), y una mediana de 23 años, lo cual denota que se trataba de mujeres jóvenes. La tabla 1 muestra las frecuencias de agentes 
para este grupo. Además, en el 11,6 \% de los casos se observaron diplococos gram negativos intracelulares y extracelulares con una reacción leucocitaria aumentada compatible con uretritis gonocócica. De igual modo, en esta población se observaron coinfecciones polimicrobianas entre $C$. trachomatis y $N$. gonorrhoeae las (2,9 \%), G vaginalis y C. trachomatis (1,4 \%), y G. vaginalis, T. vaginalis y $C$. albicans $(1,4 \%)$. El método molecular AMPLICOR ${ }^{\circledR}$ CT/NG permitió establecer que 15,9 \% y 4,3\% de la población de alto riesgo presenta infección por C. trachomatis y $N$. gonorrhoeae respectivamente.

La población de bajo riesgo $(\mathrm{n}=16)$ presentó una edad promedio de 28,5 años y una mediana de 26 años. La tabla 1 muestra las frecuencias de infecciones para esta población. El patrón de coinfecciones polimicrobianas fue $C$. trachomatis y Gr vaginalis (12,5\%), G. vaginalis y $N$. gonorrhoeae (6,2 $\%)$, y G. vaginalis y C. albicans (6,2 \%). En esta población el método molecular aplicado permitió diagnosticar infección por $C$. trachomatis y $N$. gonorrhoeae en un $12,5 \%$ y $\quad 6,3 \%$ respectivamente.

Tabla 1. Frecuencia de agentes causales de ITS por medio de protocolos microbiológicos estándares, método molecular y según población de riesgo,

\begin{tabular}{|c|c|c|c|}
\hline Población & Agente & Positivo & $\%$ \\
\hline \multirow{6}{*}{$\begin{array}{c}\text { Alto Riesgo } \\
n=69\end{array}$} & Gardenella & 12 & 17,4 \\
\hline & Tricomonas & 2 & 2,9 \\
\hline & N. gonorrhoeae & 15 & 21,7 \\
\hline & Candida albicans & 2 & 2,9 \\
\hline & PCR - NG & 3 & 4,3 \\
\hline & PCR - CT & 11 & 15,9 \\
\hline \multirow{6}{*}{$\begin{array}{c}\text { Bajo Riesgo } \\
n=16\end{array}$} & Gardenella & 9 & 56,3 \\
\hline & Tricomonas & 0 & 0,0 \\
\hline & N. gonorrhoeae & 0 & 0,0 \\
\hline & Candida albicans & 2 & 12,5 \\
\hline & PCR - NG & 1 & 6,3 \\
\hline & PCR - CT & 2 & 12,5 \\
\hline
\end{tabular}

Factores de riesgo asociados a las ITS

Antecedentes de ITS. Un 4,4 \% de la población de alto riesgo refirió presencia y recurrencia de enfermedades como gonorrea y herpes las cuales no se refirieron en la población de bajo riesgo. 
Uso de sustancias antimicrobianas. El $55 \%$ del grupo de alto riesgo manifestó utilización frecuente de sustancias antimicrobianas como lavados, cremas u óvulos vaginales frente a un $45 \%$ en el grupo de bajo riesgo.

Numero de compañeros sexuales: En el estudio se determinó que la población de trabajadoras sexuales tiene una frecuencia de relaciones sexuales que oscilan entre 5-20 por semanas. De las cuales 70 \% tiene una frecuencia entre 5-10 por semana, $10 \%$ de $11-15$ por semana y $20 \%$ de $16-20$ por semana. Lo cual permitió estimar una frecuencia de relaciones sexuales anuales de más de 1 000 en por lo menos el 20\% de la población. En la población de bajo riesgo se determinó que todas tenían un solo compañero sexual estable.

Uso de preservativos. El 15,4 \% de las trabajadoras sexuales no utilizan preservativos en todas sus relaciones sexuales y el resto al parecer si (84, 6 $\%)$ ya sea en su trabajo o fuera de este. Para el caso de la población de bajo riesgo se determinó que ninguna lo utilizaba.

\section{DISCUSIÓN}

Este es el primer estudio realizado en el Departamento de Córdoba, que permitió identificar los principales agentes etiológicos específicos de las enfermedades de transmisión sexual. Las trabajadoras sexuales son unos de los grupos claves para la transmisión de las ITS y del HIV, siendo el grupo enlace o centinela con la población general para prevenir el HIV y las ITS. Es necesario conocer la prevalencia de las ITS más comunes tales como la infección por clamidia y gonococo, para diseñar programas eficaces en la contención de las ITS. El control de las ITS entre las trabajadoras sexuales es crucial ya que es un indicador estimativo de los costos económicos que ocasionan los tratamientos de las ITS tanto en los grupos de alto como de bajo riesgo. Esto es importante en especial en edades productivas como la de la población de este estudio que fue similar a las encontradas en otros países (3,11-13,16-18).

La prevalencia de ITS encontrada en este estudio en población de trabajadoras sexuales, coincide con otros estudios en EEUU en poblaciones aparentes de bajo riesgo (19). En éste tuvieron tasas de ataque para CT en Seattle (8,7 \%), Indianápolis (15,3 \%) y New Orleáns (10,1 \%) en una población que no eran trabajadoras sexuales. Estas tasas son similares a las encontradas en nuestro estudio que a pesar de analizar una casuística baja, encontró en trabajadoras sexuales un 15,9 \% lo que demuestra que las ITS por CT no son exclusivas de una determinada población. En Lisboa (20) en una población de 
211 reclusas, se encontró un 3,6 \% de infección por CT y una prevalencia total de otros gérmenes del $87 \%$. En este grupo portugués la vaginosis (37\%) al igual que nuestro estudio (17,4 \%), fue el trastorno más común encontrado. En Bangladesh (10), en una población de 400 trabajadoras sexuales se encontraron prevalencia de $36 \%$ para $N$. gonorrhoeae (NG) y 43,5 \% para CT, cifras similares a los estudios asiáticos y africanos. Se calcula que en Bangladesh existen entre 50 a 100 mil trabajadoras sexuales (10), al igual que en Colombia son cifras inexactas, ya que existe un subregistro de hoteles y casas que sirven de prostíbulos.

Regionalmente (12), en Alagoas (Brasil), un Estado de condiciones sociodemográficas similares al departamento de Córdoba, se encontraron prevalencias para NG y CT del 6,4\%, en poblaciones femeninas rurales de aparente bajo riesgo pero al igual que la nuestra de escasos recursos económicos y bajo nivel educativo. En nuestro trabajo se hallaron tasas mayores de infección para CT (15,9\%) y NG (11,6 \%), pero fueron mas baja para Trichomonas en el nuestro (10,3 \% vs 2,9 \%). En Bogotá Molano et al(21) en una población de bajo estrato socio-económico, hallaron para CT una prevalencia de un $5 \%$, sin embargo, la población fue de bajo riesgo, en contraste con nuestro estudio y el portugués (20).

Las coinfecciones polimicrobianas estuvieron presentes como se esperaba en las ITS y especialmente en los grupos de riesgo $(10,11,13,17)$. La asociación de clamidia y gonococos con otros patógenos indican la gran cantidad de parejas sexuales del grupo de estudio. En Brasil (12) en una población de bajo riesgo un $51 \%$ de las mujeres estudiadas tenían múltiples infecciones por diferentes gérmenes.

Existen estudios que indican que tener más de un compañero sexual en 60 días se asocian significativamente al incremento de la infección por clamidia y por gonococos (3,5,8,13,17,20,22-25). En nuestro trabajo fue dramáticamente alto el número de relaciones sexuales referidas con un promedio entre 5-20 por semana, esto indica que la población de trabajadoras sexuales de Montería, tendría unas 720 relaciones anuales por persona. Esto demuestra las altas tasas de infección y de asociación entre microorganismos encontrados. En la población estudiada estos gérmenes, son un marcador o cofactor de que existan otras ITS como HIV, sífilis, HPV y hepatitis, lo que justifica plenamente la rápida implementación de un programa para la prevención y control de ITS en las trabajadoras sexuales de Montería. 
Es importante recordar, el papel de CT como agente "marcador" de ITS reconocido mundialmente (25). OMS (1) estimo en el 2001 aparecieron 92 millones de casos nuevos debido a la infección por este germen. De otro lado, se cree que las infecciones por CT facilitan la transmisión de HIV $(9-11,25)$ lo que refuerza la importancia de nuestro trabajo.

A pesar del alto uso del condón reportado en este estudio (84,6\%), la prevalencia de ITS fue alta. En ese sentido, el uso del condón en Seattle (19), fue asociado con una baja prevalencia de ITS, sin embargo, no fue así en las otras ciudades estudiadas como Indianápolis y New Orleans (19). En nuestro estudio a pesar del uso del condón, se encontraron altas tasas de infección lo que demuestra que algo sesgó la encuesta. La población de estudio es altamente vulnerable, no tienen seguridad social ni servicios médicos, su estatus laboral ilegal pero a la vez consentido por el Estado, las coloca en situaciones sicológicas contradictorias y a la vez peligrosa. Por ello es muy probable que las encuestadas mintieran para obtener su permiso de ejercer el trabajo sexual, ya que la entrevista se llevo a cabo en el centro de atención para trabajadores sexuales, y en donde se les expide "permiso" para ejercer la prostitución. También es posible que ellas no se protejan con su compañero estable, y que este ultimo tampoco lo haga en su vida extramarital como se ha demostrado en otros trabajos similares en Asia y Africa $(11,12,17,25)$.

Los aspectos socio-culturales y el grado de escolaridad son determinantes en la conducta sexual y en el riesgo de contraer ITS. Si se tiene en cuenta este aspecto se pueden identificar factores de riesgo para ser usados en estos grupos específicos en programas de intervención para cambiar el comportamiento sexual de estas personas. Nuestra población de trabajadoras sexuales tenían un bajo grado de escolaridad, escasos recursos económicos y muchas de ellas con historia de violencia intrafamiliar y sexual (datos no mostrados). Estos factores han sido recientemente reportados en la conferencia mundial sobre enfermedades infecciosas de la mujer donde se evidencio la alta vulnerabilidad de adquirir una ITS por parte de las poblaciones femeninas $(2,4)$.

De otro lado, el uso de las técnicas de diagnostico molecular demostró su importancia en este tipo de estudios. Es difícil aislar NG y en el grupo de trabajadoras sexuales estudiadas se reporto la utilización de antisépticos vaginales en un $55 \%$ con lo que se dificulto el aislamiento. Se encontró un alto porcentaje de CT (15,9\%) y de NG (4,34 \%), en otros estudios en diferentes países con técnicas moleculares similares a las utilizadas por nosotros también demostraron su utilidad $(13,19,16)$. 
De otra parte, este estudio incluyo un grupo testigo de mujeres con conductas sexuales de bajo riesgo y sin antecedentes de ITS. Aunque el grupo estuvo conformado por solo 16 mujeres, se obtuvieron resultados interesantes, como la alta tasa de infección por $G$ vaginalis (56,3 \%), NG (6,3 \%), mas altas que las del grupo de riesgo 17,4\% y 4,3\% respectivamente. Obviamente es sesgado comparar tasas de infección en poblaciones con diferencias en el tamaño de la muestra. Sin embargo, es preocupante ya que algo sucedió en la encuesta, o existe algún grado de promiscuidad no detectado en la entrevista debido a que el compañero o esposo no pudo ser entrevistado. En este tipo de poblaciones también hay que intervenir con profundos y serios programas educativos, este grupo testigo también poseían un bajo nivel socio-educativo, y en estos conglomerados la mujer también es muy vulnerable y se deben llevar a cabo campañas tendientes a evitar la propagación del HIV y de otras ITS.

La asociación del HIV y las ITS permiten desarrollar estrategias comunes de control para estas patologías. La prevalencia y la incidencia de algunas ITS cambian dramáticamente y pueden ser usadas como un marcador para establecer los cambios en las conductas sexuales y por lo tanto la incidencia de HIV (23-25). La alta prevalencia de CT, NG y otros agentes etiológicos de ITS encontrados en este estudio, refuerza la necesidad de ahondar en el estudio completo de otras etiologías, como HPV, hepatitis B, C, sífilis y Haemophilus ducreyii, entre otros

Agradecimientos. A la Universidad de Córdoba y al Comité Central de investigaciones (CIUC) por el apoyo económico a este proyecto. A la secretaría de salud municipal de la ciudad de Montería por avalar este estudio.

\section{REFERENCIAS}

1. Organización Mundial de la Salud/Organización Panamericana de la Salud. Pautas para la vigilancia de las infecciones de transmisión sexual, WHO/CHS/ HSI/ 99.2. WHO/CDS/CSR/EDC/99.3 UNAIDS/00.03S; 1999.

2. Periago MR, Fescina R, Ramon P. Steps for preventing infectious diseases in women. Emerg Infect Dis 2004; 10: 1968-73.

3. CDC Surveillance Data, Division of STD Prevention (DSTD), National Center for HIV, STD, and TB Prevention (NCHSTP), Centers for Disease Control and Prevention (CDC), by the STD control programs and health departments; 2002.

4. McDonlad M, Anker M, Deal C, Mawle A, O'connor S, Slaughter L. International Conference on women and Infectious diseases. Emerg Infect Dis 2004;10:1963-64 
5. Ethier KA, Kershaw T, Niccolai L, Lewis JB, Ickovics JR. Adolescent women underestimate their susceptibility to sexually transmitted infections. Sex Transm Infect 2003; 79:408-411.

6. Rooney G, Daniels D, FitzGerald M, Ahmed-Jushuf I, Radcliffe K, Welch J. Specifications for the development of guidelines on the management of sexually transmitted infections and closely related conditions. International Journal of STD\& AIDS 2004; 15: 299-305.

7. U.S. Department of Health and Human Services. Healthy People 2010. 2nd ed. With Understanding and Improving Health and objectives for Improving Health. 2 vols. Washington, DC: U.S. Government Printing Office; November 2000.

8. Barros T, Barreto D, Pérez F, Santander R, Yépez E, Abad-Franch F. Aguilar M. Un modelo de prevención primaria de las enfermedades de transmisión sexual y del VIH/sida en adolescentes. Rev Panam Salud Pública 2001; 10: 89-93.

9. Frieda MT, BehITS F, Rasolofomanana JR, Van Damme K, Vaovola G, Andriamiadana J, Ranaivo J and the Mad-STI Working Group. Evidence-based treatment guidelines for sexually transmitted infections developed with and for female sex workers. Trop Med Internal Health. 2003; 8: 251-258.

10. Nessa K, Waris S-A, Sultan Z, Monira S, Hossain M, Nahar S, et al. Epidemiology and Etiology of Sexually Transmitted Infection among Hotel-Based Sex Workers in Dhaka, Bangladesh. J Clin Microbiol 2004; 42: 618-621.

11. Zachariah R, Spielmann MP, Harries AD, Nkhoma W, ChantuloA, Arendt V. Sexually transmitted infections and sexual behavior among commercial sex workers in a rural district of Malawi. International Journal of STD \& AIDS 2003; 14: 185188

12. Soares VL, Torres AM, Cavalcante GT, Silva ZP, Hora V, Diedrich T, et al. Sexually transmitted infections in a female population in rural north-east Brazil: prevalence, morbidity and risk factors. Tropical Medicine and International Health 2003; 8:595-603.

13. Orroth KK, Korenromp EL, White RG, Changalucha J, de Vlas SJ, GrayR H, et al. Comparison of STD prevalences in the Mwanza, Rakai, and Masaka trial populations: the role of selection bias and diagnostic errors. Sex Transm Infect 2003; 79: 98-105.

14. Janda W, Knapp J. Neisseria and Moraxella catarralis. In: Murray M(Ed). Manual of clinical Microbiology, 8th edition. Washington, USA: ASM Press; 2003. pp. 585-600.

15. World Medical Association declaration of Helsinki. Ethical Principles for Medical Research Involving Human Subjects, WMA General Assembly, Tokio, October 2004 [Internet]. Disponible en: http://www.wma.net/e/policy/b3.htm Consultado: Diciembre de 2005. 
16. Williams H, Tabrizi SN, Lee W, Kovacs GT, Garland S. Adolescence and other risk factors for Chlamydia trachomatis genitourinary infection in women in Melbourne, Australia. Sex Transm Infect 2003; 79:31-34.

17. Wong ML, Lubek I, Dy BC, Pen S, Kros S, Chhit M. Social and behavioral factors associated with condom use among direct sex workers in Siam Rep, Cambodia. Sex Transm Infect 2003; 79:163-165.

18. Aldeen T, Haghdoost A, Hay P. Urine based screening for asymptomatic/ undiagnosed genital chlamydial infection in young people visiting the accident and emergency department is feasible, acceptable, and can be epidemiologically helpful. Sex Transm Infect 2003; 79:229-233.

19. Kohl KS, Sternberg MR, Markowitz LE, Blythe M, Kissinger P, Lafferty WE, et al. Screening of males for Chlamydia trachomatis and Neisseria gonorrhoeae infections at STD clinics in three US cities-Indianapolis, New Orleans, Seattle. International Journal of STD \& AIDS 2004; 15: 822-828.

20. Garcia A, Exposto F, Prieto E, Lopes M, Duarte A, Correia Da Silva R. Association of Trichomonas vaginalis with socio-demographic factors and other STDs among female inmates in Lisbon. International Journal of STD \& AIDS 2004; 15: 615-618.

21. Molano M, Weiderpass E, Posso H, Morre SA, Ronderos M, Franceschi S, et al. Prevalence and determinants of Chlamydia trachomatis infections in women from Bogotá, Colombia. Sex Transm Infect 2003; 79:474-478.

22. Pinkerton SD, Layde PM, Di Franceisco W, Chesson HW, NIMH Multisite HIV Prevention Trial Group. All STDs are not created equal: an analysis of the differential effects of sexual behaviour changes on different STDs. International Journal of STD \& AIDS 2003; 14: 320-328.

23. Gutierrez JP, Bertozzi SM, Conde-Glez CJ, Sanchez-Aleman MA. Risk behaviors of 15-21 year olds in Mexico lead to a high prevalence of sexually transmitted infections: results of a survey in disadvantaged urban areas. BMC Public Health. 2006; 6: 49.

24. Sevgi OA, Mead O, Manhart L, Colmes K. Sexually transmitted infections. Disease control Priorities in developing countries. 2ndedition. In: Jamison DT, Breman JG, Measham AR, Alleyne G, Claeson M, Evans DB, Jha P, Mills A, Musgrove P. (Editors). Washington, D.C: IBRD/The World Bank and Oxford University Press; 2006.

25. Cohen DA, FarleyTA, Mason K, Ridgeway G. The collectivity of sexual behavior. Int J STD AIDS 2006; 17:151-156. 